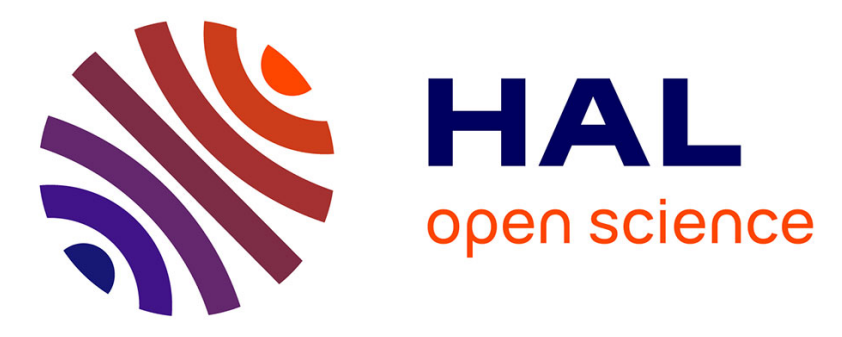

\title{
Learning to Synchronously Imitate Gestures Using Entrainment Effect
}

\author{
Eva Ansermin, Ghiles Mostafaoui, Nils Beausse, Philippe Gaussier
}

\section{To cite this version:}

Eva Ansermin, Ghiles Mostafaoui, Nils Beausse, Philippe Gaussier. Learning to Synchronously Imitate Gestures Using Entrainment Effect. Simulation of Adaptative Behaviour ( SAB), Aug 2016, Aberyswyth, United Kingdom. pp.219 - 231, 10.1007/978-3-319-43488-9_20 . hal-01377250

\section{HAL Id: hal-01377250 \\ https://hal.science/hal-01377250}

Submitted on 6 Oct 2016

HAL is a multi-disciplinary open access archive for the deposit and dissemination of scientific research documents, whether they are published or not. The documents may come from teaching and research institutions in France or abroad, or from public or private research centers.
L'archive ouverte pluridisciplinaire HAL, est destinée au dépôt et à la diffusion de documents scientifiques de niveau recherche, publiés ou non, émanant des établissements d'enseignement et de recherche français ou étrangers, des laboratoires publics ou privés.

$$
\text { Copyright }
$$




\title{
Learning to synchronously imitate gestures using entrainment effect
}

\author{
Eva Ansermin, Ghiles Mostafaoui, Nils Beausse, and Philippe Gaussier \\ Neurocybernetic team, ETIS laboratory, \\ CNRS, ENSEA, University of Cergy-Pontoise, 95302 France, work funded by the DGA and the \\ DIRAC project. \{eva.ansermin, ghiles.mostafaoui, nils.beausse, \\ philippe.gaussier\}@ensea.fr \\ www-etis.ensea.fr
}

\begin{abstract}
Synchronisation and coordination are omnipresent and essential in humans interactions. Because of their unavoidable and unintentional aspect, those phenomena could be the consequences of a low level mechanism: a driving force originating from external stimuli called the entrainment effect. In the light of its importance in interaction and wishing to define new HRI, we suggest to model this entrainment to highlight its efficiency for gesture learning during imitative games and for reducing the computational complexity. We will put forward the capacity of adaptation offered by the entrainment effect. Hence, we present in this paper a neural model for gesture learning by imitation using entrainment effect applied to a NAO robot interacting with a human partner.
\end{abstract}

Keywords: Entrainment, Synchrony, gesture learning, Human Robot Interaction, Neural Network

\section{Introduction}

Humans tend to be set in motion by strong or rhythmical stimuli [12]. This driving force, which allows us to be reactive and adaptive, is called the entrainment effect. This phenomenon, also called magnet effect, is strongly linked to our ability to be synchronized and coordinated with external stimuli. Under some conditions, one can consider that synchrony is caused by the entrainment effect. For example, a synchronous interaction between two partners can be seen as the result of a mutual and bi-directional entrainment. In fact, entrainment can be observed with different modalities in various human-human interactions and plays an obvious role in social coordination (walking together, playing music, dancing, imitating etc.)[18]. This influence on our motor control have been largely analysed by psychological studies about interpersonal coordination. Varlet et al [21] revealed that the continuity of the stimuli rhythms has a fundamental role in influencing the visual and auditory motor coordination, Lagarde and Kelso [15] found similar results by studying the multi-modal coordination dynamics between the senses (sound and touch) and human movements.

Another interesting characteristic of entrainment and synchrony is their unintentional aspect. Indeed, we can distinguish intended and unintended synchrony. In the first case, synchronisation is aimed whether in the second, it is spontaneous and occurs 
without the subject noticing. Numerous researches found out that two subjects interacting with each other tend to unintentionally synchronise [10]. This mutual unintended convergence toward a similar interacting rhythm can occur when the subject's own frequencies are close (supposedly a difference of more or less $10 \%$ ), otherwise, it may be difficult for the interacting partners to be "unintentionally" synchronized. Nevertheless, even in this case one can notice the presence of mutual unintentional entrainment (each partner driving the other toward his own motion dynamic)[8]. In fact, as demonstrated in [10], this phenomenon is such that unintentional entrainment cannot be willingly avoid. It is precisely this unavoidable aspect which makes the entrainment effect very interesting to model for Human-Robot Interactions (HRI). We believe that rhythmic adaptation does not only sustain the interaction but also is caused by the interaction through mutual entrainment.

More precisely, in this paper we address the question of integrating the so called entrainment (or magnet effect) in a neural model for HRI. Indeed, despite its importance in social interactions, this phenomenon is seldom taken into account when modelling those interactions. Yet, as we will show in this study, modelling the entrainment effect can make human/robot interacting tasks easier thanks to the adaptability its offers. This aspect will be highlighted through an experimental study presenting an example of a neural network model based a on low level entrainment and designed for learning gestures during an imitative games between a human and a NAO robot. We will demonstrate that integrating the entrainment effect simplifies drastically the computational complexity.

\section{Related Works and Positioning}

Broaching the subject of interpersonal coordination (entrainment, synchronisation) during interactions involves addressing the issue of gaining sensory motor abilities to be able to adapt our motion dynamics and behaviour according to external stimuli.

A classical way to approach this question is to consider a sensory motor system capable of predicting and adapting its behaviour after analysing the observed stimuli. Several efficient bio-inspired computational models have been proposed in this line. As examples, Demiris et al performed experiments in which a robotic head equipped with a pair of cameras observed and imitated the head movements of a human demonstrator [4], Blanchard and Canamero proposed the basis of a simple algorithm generating explorative and imitative behaviours [2], Jenkins et al. described an imitation model based on a set of perceptuo-motor primitives. A simple version of the model was validated on a 20 DOF simulated humanoid using real vision data to imitate movements from athletics and dance [11].

Despite their promising results, those approaches imply a relative high level of processing (observing, analysing, predicting and adapting at each time) which does not explain the unintentional aspect of entrainment and synchronization in human-human interactions. In fact, recent works of Dumas et al. using hyper-scanning has revealed the emergence of millisecond inter-brain synchronization across multiple frequencies bands during social interactions (spontaneous exchanges between two participants of intransitive bi-manual movements [5]). Moreover, Varlet et al. investigated social mo- 
tor coordination of patients suffering from schizophrenia. The results demonstrated that patients intentional motor coordination was altered while their unintentional low level motor coordination was retained implying that unintentional and intentional coordination are not part of the same process [22]. We can conclude that these inter subject synchronizations are not planned as high level processing but result in low level analogical synchronization of neural populations from the sensory flow (vision, audition...). Otherwise millisecond synchronization would not be obtained. Another way to model and explain interpersonal coordination is to considered the two interacting agents as $d y$ namical systems influencing each other. Their behaviours can be considered as Hugens metronomes [17] cross influencing each other in a "mechanical way" via several signals (audition, vision etc.). In this case, subjects (or their limbs) can be modelled by oscillatory systems entraining each other. This mutual driving force can lead to synchronisation. Hence synchronisation would only be a particular case where the entrainment effect is strong enough (depending on the range between the partner frequencies) to reach a stable convergent state where the frequencies of the partners are equal and in phase. This type of approaches is clearly a better way to explain human tendencies to be unintentionally and unavoidably entrained by others without noticing or without "predicting" it.

\subsection{Modelling sensori-motor coordination in dynamical systems}

As previously mentioned, the interacting partners behaviours are modelled by rhythmic or oscillatory systems in a dynamical system approach. In other words, the motor controllers of the partner s body parts are often described as a set of oscillators. This way to define motor controllers is inspired by the fact that body parts can be seen as oscillatory systems (pendulums for example) due to their physiognomy and capacity of movement [7]. Furthermore, several neuro-biological studies highlighted the presence of a strong oscillatory component in human and animal motor control. In fact, researches on the locomotion of several species has allowed to put forward the existence of a neural network located in the spine and enabling a minimal rhythmic autonomous motor control [6]. This set of oscillators is called Central Pattern Generator (CPG) and is supposed to be involved in several task such as breathing, eating or walking. Other recent studies suggest that motor cortex responses during non oscillatory movements (reaching) contain a brief but strong oscillatory component [3]. For those reasons, oscillators are often used to define the motor behaviour of interacting agents in a dynamical system approach.

To model the mutual exchange of informations (entrainment) between the interacting systems (agents) those oscillators are often coupled in a non linear way as in the well known Haken Kelso Bunz model (HKB) [13] where a Van Der Pol oscillator is coupled with a Rayleigh oscillator. This mathematical model permits to report the effect of using the energy of an oscillator to entrain the other. From a neural point of view, oscillators are often modelled as two linked neurons which inhibit and stimulate each other to maintain an oscillatory behaviour. The way they are linked and the behaviour of the neurons are depending on the type of oscillators: Wilson- Cowan [23], Terman-Wang [20], Revel [19]etc. Indeed echo state networks are used to get a complex 
response to a precise entry. Thus, rhythmical complex and adaptive movements can be obtained from such networks [16].

\subsection{Positioning on entrainment model and its advantage}

Being interested in modelling entrainment effects for HRI, we will adopt an approach using dynamical systems as this is an efficient way to describe the possible unconscious or unpredictable aspects of this low level phenomenon (see above for the justifications). The models based on dynamical systems theories presented above are effective but possess some limitations because of their computational complexity or the obligation to have access to the parameters of both oscillators (agents motor controllers) which is not always possible if we consider the practical case of a robot interacting with a human.

In our previous works, we proposed a solution to overcome those limitations by endowing a NAO robot with a neural model which uses the energy of the optical flow (visual stimuli) induced by the human partner movements to "directly" entrain the robot's motor controller [9]. This model, which permits to modify the dynamic of a given movement, was tested and validated with one oscillator as a motor controller and a very simple gesture (one arm moving up and down) and will be more precisely described below section 5.2. Here we propose the same approach using this entrainment model to study its possible use for learning (by imitating) more complex gestures and trajectories. We will prove that the use of entrainment in the neural model proposed in this paper can not only enhance the adaptability of a humanoid robot interacting with a human but also simplifies the computational complexity.

\section{System workflow and Experimental setup}

We used a minimal set-up for our experiments as shown in figure 1. The components include a Nao robot, an external camera to avoid the limitations of NAOs camera and

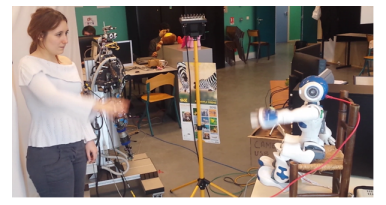
a human partner. The frame rate is 30 images per second. During the experiments, the human partner faces the robot as shown in figure 1 and moves rhythmically his arm. Our objective is to build a model based on entrainment effect in order to give the robot the ability to imitate synchroniously the human gesture. Only the NAO's shoulder articulation is

Fig. 1: Experimental setup: Exemple of an imitation game between NAO and a human partner used and controlled by the model ( 2 degrees of freedom: up and down/left and right). As we wish the robot to be able to reproduce a rhythmical movement shown by an interacting partner, the NAO's motor controller will be generated by a reservoir of oscillators (see figure 2). The idea is to define the desired motor signal as a weighted sum of oscillators at different frequencies (such as in Fourier series). Hence reproducing a movement or a trajectory means finding the right combination within a set of oscillators. The general workflow of our model, illustrated figure 2, can be summarized as follow: 


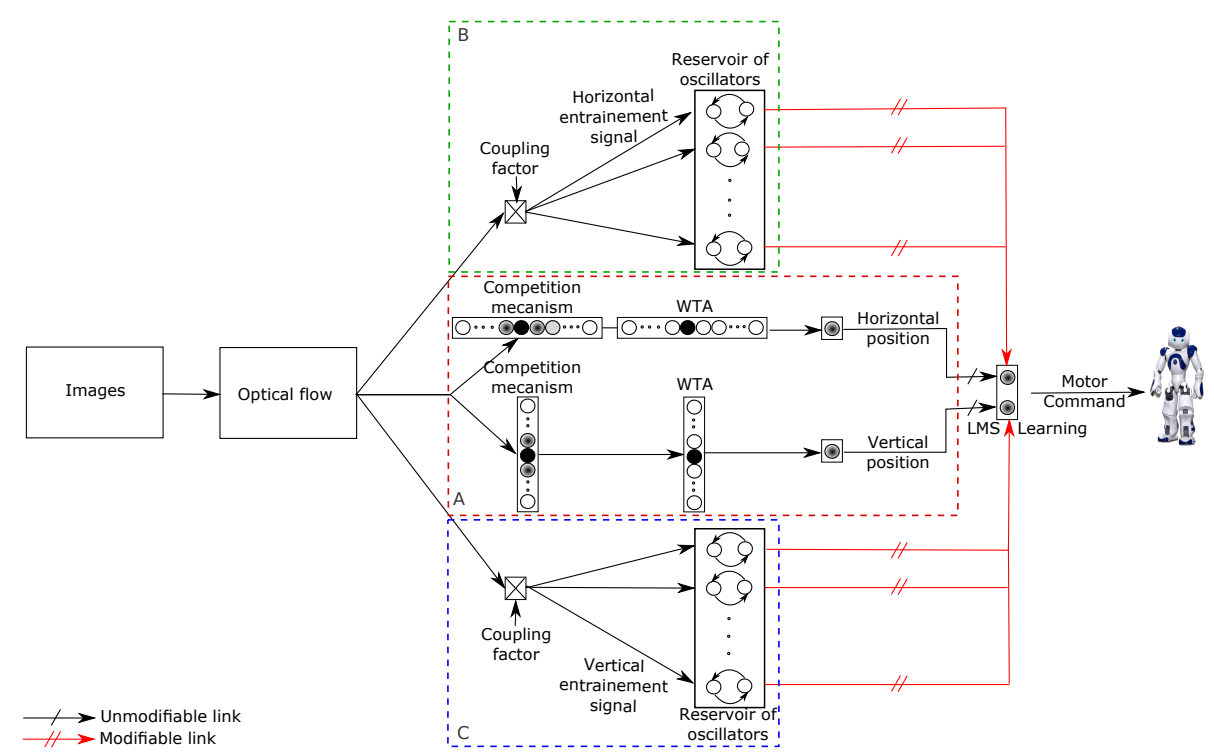

Fig. 2: Structure of the imitation model

- The images of the camera capturing the human movements are used to compute the optical flow

- The so resulted optical flow is then used to:

- Extract the $X$ and $Y$ coordinates of the human movement trajectory (A block in figure 2).

- Build the the $X$ entrainment signal (B block in figure 2) and the $Y$ entrainment signal (C block in figure 2) which will influence (entrain) and modify the frequencies and phases of the oscillators in the reservoir which commands the motor controller of the robot.

- By modifying the weight of each oscillator of the reservoir, the Least Mean Square (LMS) algorithm will learn the combination of oscillators describing the $X$ and $Y$ desired trajectory extracted from the human motion by the A block.

- Finally, the outputs of the two LMS neurons are used directly as the final motor controller signals leading the robot to imitate the human 's gestures.

Each part of the model will now be detailed in sections below.

\section{Extracting the trajectories to imitate}

As mentioned above, the robot aims at imitating the gestures of the human partner. The trajectory to learn corresponds consequently to the one described by the human 's moving arm, and more precisely by the human 's hand (see the experimental setup section 3). Rather than performing a complex image processing to recognize and localize the moving hand, we based upon the fact that this hand is the body part which 
moves the most comparing to the rest of the arm. Consequently, the trajectory to learn and imitate will be defined, at each time, by the $x$ and $y$ coordinates of the point in the image having the higher optical flow. To do so, we first measure the optical flow using the hierarchical algorithm described by Amiaz et al [1]. At each time $t$, the optical flow is computed for all the image pixels and projected to a set of 240 neurons, 120 for the horizontal component and 120 for the vertical one. In order to filter the noise, a simple competition mechanism is used. Each neuron is stimulated by its closest neighbours and inhibited by the distant neurons. This process is equivalent to a convolution by a Difference of two Gaussians filter highlighting consequently the local maxima of the optical flow by taking into account its local distribution. A Winner Takes All (WTA) is then used to extract the $x$ and $y$ coordinates of the point (with a higher filtered optical flow) describing the human hand trajectory to imitate (see A block in figure 2). Two examples of extracted trajectories are illustrated in figure 6 in the case of a human moving his hand (circular and infinite shape movement).

\section{Modelling the entrainement effect}

\subsection{The reservoir of oscillators}

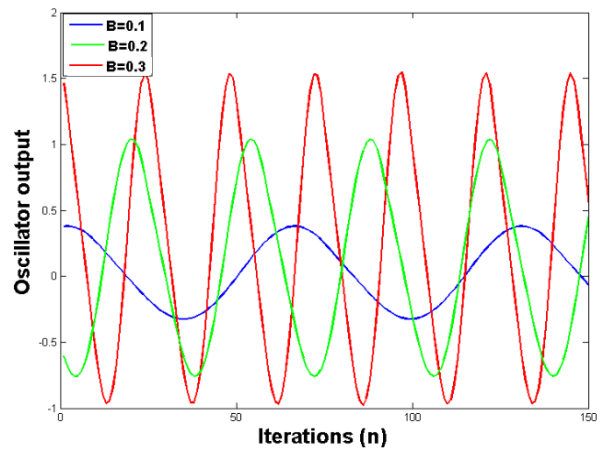

Fig. 3: 3 Oscillator outputs for different values of $\beta$

The resulting signals are defined by the equations below:

$$
\begin{aligned}
& N_{1}(n+1)=N_{1}(n)-\beta N_{2}(n)+\alpha 1 \\
& N_{2}(n+1)=N_{1}(n)+\beta N_{2}(n)+\alpha 2
\end{aligned}
$$

By modifying the $\beta$ parameter, we defined our two sets of 8 oscillators whose own frequencies are between $0.5 \mathrm{~Hz}$ and $2.6 \mathrm{~Hz}$. We heuristically choose these frequencies 
to cover a large range of possible rhythms reachable by the human and mechanically plausible for the NAO. Figure 3 illustrates the oscillatory signals obtained for three different values of $\beta$.

\subsection{The entrainement model}

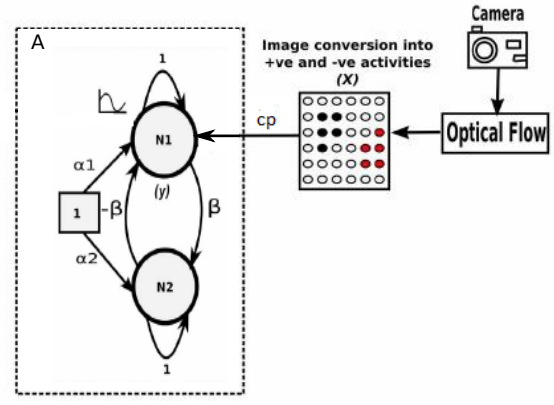

Fig. 4: Hasnain et al entrainment model
As previously detailed, the usual methods to model entrainment impose to have access to both oscillating systems. To avoid this limitations, we proposed in our previous works a simple neural model where the interacting agent (a robot and a human) are coupled via low level visual information. For clarity sake, this model will be summarized in this section but we invite the reader to refer to [9] for a detailed description. We took inspiration from interpersonal coordination studies demonstrating that unintended motor entrainment can not be avoided (see section 3). This observation implies a strong and direct link between the external stimuli and the motor controller. Starting from these conclusions, the model presented in [9] and illustrated in figure 4 propose to use the energy induced by the optical flow of the human partner movement to entrain the robot motor controller. The oscillatory signal controlling the robot's arm (equation 1) can be rewritten as fellow (equation 3 ):

$$
N 1(n+1)=N 1(n)-\beta N 2(n)-\alpha 1+c p * f(n)
$$

with $f(n)$ the entrainment signal and $c p$ the coupling factor. $f(n)$ is deducted at each time by a spatial integration of the optical flow. As the optical flow can be either positive or negative according to the movement direction, $f(n)$ oscillates in the case of rhythmic movements. This resulted signal is modulated by a coupling factor $c p$ (see figure 4) and added to the oscillator to modify its dynamic and hence the robot behavior. The coupling factor $c p$ is included into 0 to 1 , it allows to modulate the energy brought in the oscillator: the higher it is, the more important the entrainment is. This implies that the range of frequencies in which the oscillators are able to synchronise can change according to $c p$ and be larger for a higher coupling factor. Using this model we demonstrated that a synchronous interaction can emerge considering the fact that humans will also be entrained by the modifications of the robot 's behaviours. Those results were validated by experimental studies in psychology with naive subjects [8]. 


\section{Learning by imitating}

To learn the desired movement sequence and to decompose it in an oscillatory base, we use a Least Mean Square algorithm network (see figure 2). This algorithm is a supervised learning where we aim at reducing the square error between a required sequence and the different available input signals whose weight can be modified (cf figure 5). At each iteration, the algorithm compares the error with the precedent and changes the weights in function. Those modifications are made according to the following equations:

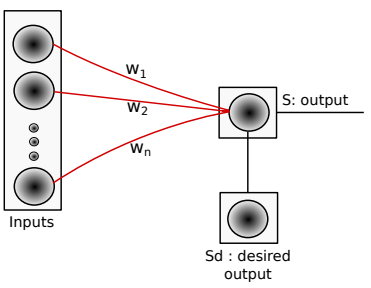

$$
\left\{\begin{array}{r}
S(n)=\sum_{i=1}^{p}\left(w_{i} * b_{i}\right) \\
W_{i}(n+1)=W_{i}(n)-\varepsilon \Delta e(n) \\
e(n)=(S d-S)^{2}
\end{array}\right.
$$

Fig. 5: Structure of the LMS Network

with:

- $\varepsilon$ the learning step. A higher $\varepsilon$ permits to modify the weights quickly but makes it more sensitive to noise. $\varepsilon$ is set to a fixed value of 0.1 here.

- $S d$ an $S$ respectively the desired signal to learn and the output of the LMS.

With this model we try to learn a rhythmical motion sequence. As we wish to reproduce any rhythmical signal and because of the nature of the algorithm, the learning is possible only if we can find a set of oscillators with adequate frequencies and phases to describe the desired signal. In this context, this implies to have a very high number of oscillators. To resolve this problematic we will use the entrainment model presented earlier to change the set of oscillators behaviours (amplitude, frequency and phase) to better fit the desired trajectories without adding computational complexity.

Let's consider now the complete model as presented figure 2. We add the entrainment effect (presented in the previous section) to our reservoir of oscillators (part B and $\mathrm{C}$ of the figure 2).

The oscillators will now be entrained by the optical flow induced by the human movements in both $x$ and $y$ directions (respectively B and C paths in figure 2). We distinguish the horizontal and vertical component of the movement for entrainment because different studies suggest that visual entrainment can be segmented into several directions. For example, Kilner et al. showed that we tend to be entrained vertically by a vertical stimuli when making an horizontal movement [14].

The model is tested in real conditions to make the NAO robot imitate the human performing two different gestures: a circle and an infinite shape trajectory. It is worth noticing that the set of initial oscillators and the parameters used are exactly the same for the two conditions. Figure 6 illustrates the efficiency of this simple model. The robot 
is able to imitate the two different gestures after less then 30 seconds of learning thanks to the entrainment effect which facilitate the adaptation in amplitude, frequency and phase of the initial oscillators. Videos of these experiments can be seen here ${ }^{1}$.
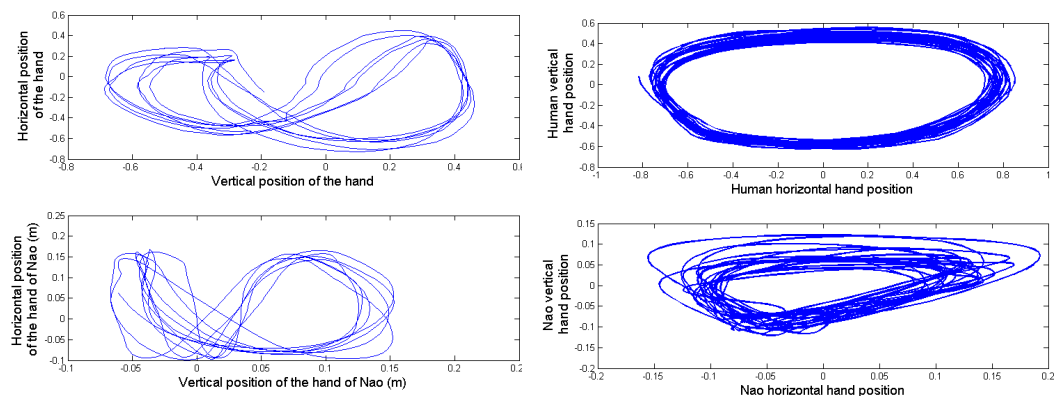

Fig. 6: Human and Robot hand trajectories during learning a round and an infinite gesture during imitation games

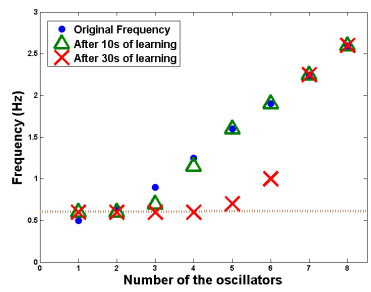

Fig. 7: Frequency of the oscillators before and after $10 \mathrm{~s}$ and 30 s learning of a $0.6 \mathrm{~Hz}$ sinus.
To clarify this mechanism, we consider the learning of a simple simulated sinus of $0.6 \mathrm{~Hz}$. The added entrainment effect permits a quick convergence of some of the oscillators to the frequencies sought by the signal to learn $(0.6 \mathrm{~Hz})$. This fast adaptation of the reservoir of oscillators to the desired signal proves the fact that using entrainment can avoid the complex problem of defining a too large number of oscillators at different frequencies and phases. The figure 7 allows us to observe the frequency after 10s and 30s of learning and put forward the fact that the oscillators do not synchronise at once and that the learning is consequently progressive.

The coupling factor can modify the range of frequencies we can define with our reservoir of oscillators. Higher values of $c p$ permit a bigger influence of the entrainment signal (optical flow of the moving partner) on the oscillators which can lead to drive them to farthest frequencies and phases (comparing to their initial status).

\subsection{Influence of the coupling factor}

\footnotetext{
${ }^{1}$ www.etis.ensea.fr/neurocyber/Videos/authors/ansermin/sab2016
} 


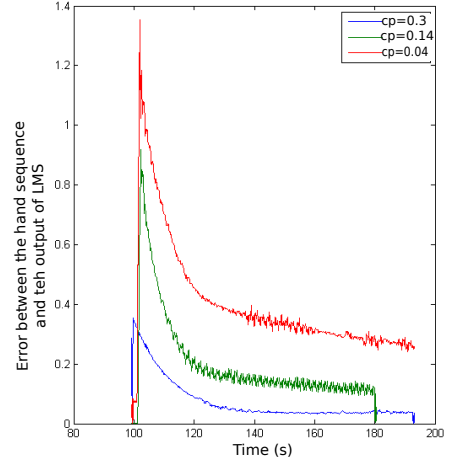

Fig. 8: Influence of the coupling factor on the convergence speed.
Consequently, the results and the convergence speed of the LMS algorithm are dependent on the $c p$ value. The figure 8 shows the reconstruction error computed by the LMS for different values of $c p$ while learning a sinus of $1 \mathrm{~Hz}$. We can observe that indeed, a higher $c p$ means a quicker learning but also more oscillators synchronised on the same harmonic of the signal as shown in figure 9 . Nevertheless, a high value of $c p$ can lead to an exaggerated entrainment which implies several synchronized oscillators (toward the fundamental frequency) leading to a synchronized behaviour but a less defined reconstruction of the desired trajectories (less harmonics). This phenomenon is illustrated in figure 10 where the simulated trajectory to learn is better explained (after learning) with a lower coupling factor.

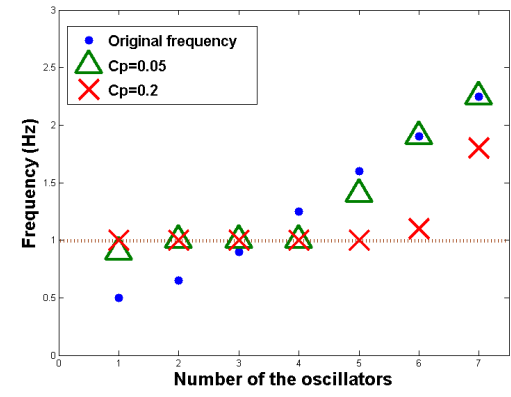

Fig. 9: Observation of the frequencies of the oscillators during the learning of an $1 \mathrm{~Hz}$ sinus for different coupling factors.

\section{Conclusion}

The entrainment effect has been proven to be very present in human behaviour during our interaction with the environment or in social interplays where it plays an important role in interpersonal coordination. Yet, it has been rather neglected by many research which tried to model interactions in HRI. In this paper we presented a model based upon low level entrainment using visual stimuli (optical flow). We proved with both 


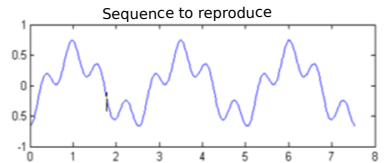
Output of LMS for $\mathrm{CP}=0.05$

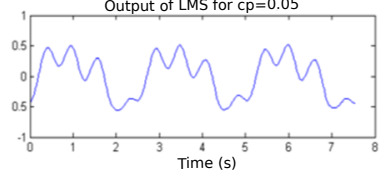

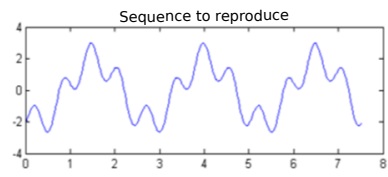

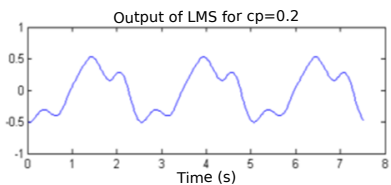

Fig. 10: Learning of a complex simulated signal (sum of 3 sinus) for different coupling factors

simulated and real experimental tests, that despite its relative simplicity, our model is able to give the robot abilities to imitate synchronously different gestures in real time while using exactly the same parameters.

Yet, this model presents some limits. Indeed, the system is unable to perform the learned movement alone, without entrainment effect. When there is no entrainment, the oscillators will return to their original frequency. Thus, if more than one oscillator have been synchronised with a harmonic of the signal and learned by the LMS, the learned signal would be deformed without the entrainment. Moreover, the phase between the different part of the movement (vertical and horizontal) cannot be maintained without entrainment. The questions of how memorizing the gestures and what information to memorize (the entrainment signal? the oscillator weights? etc.) are our near future perspectives. It is worth noticing that the principal limitations of the model is in its definition itself. In fact our objective here is to prove the efficiency of using low level entrainment of the motor controller (by the external visual stimuli) to adapt the behaviour and synchronize it with the interacting partner. We argue that learning to imitate or reproduce more complex, refined and non rhythmic gesture sequences needs a higher level of treatment including a more "predictive" aspects.

Nevertheless, regarding the fact that the entrainment was proved to be unintentional in human behaviour, our position on the matter is that learning and imitating gestures imply merging a very low level sensory motor processing (entrainment) for a fast adaptation of the motions dynamics and to eases a higher level which deals with more complex sequences, refined trajectories, social contexts etc. Such an association is clearly in our perspectives.

\section{References}

1. Amiaz, T., Lubetzky, E., Kiryati, N.: Coarse to over-fine optical flow estimation. Pattern Recogn. 40(9), 2496-2503 (Sep 2007)

2. Blanchard, A., Canamero, L.: Modulation of exploratory behavior for adaptation to the context. In: Procs of Biologically Inspired Robotics Symposium 2006. pp. 131-137 (2006) 
3. Churchland, M.M., Cunningham, J.P., Kaufman, M.T., Foster, J.D., Nuyujukian, P., Ryu, S.I., Shenoy, K.V.: Neural population dynamics during reaching. Nature 487(7405), 51-56 (07 2012)

4. Demiris, J., Rougeaux, S., Hayes, G., Berthouze, L., Kuniyoshi, Y.: Deferred imitation of human head movements by an active stereo vision head. In: Robot and Human Communication, 1997. RO-MAN'97. Proceedings., 6th IEEE International Workshop on. pp. 88-93. IEEE (1997)

5. Dumas, G., Nadel, J., Soussignan, R., Martinerie, J., Garnero, L.: Inter-brain synchronization during social interaction. PLoS ONE 5, 1-10 (08 2010)

6. Grillner, Zangger: On the central generation of locomotion in the low spinal cat (1979)

7. Haken, Kelso, Bunz: a theorical model of phase transition in human hand movements. Biological Cybernetics 51 (1985)

8. Hasnain, S.K., Mostafaoui, G., Salesse, R., Marin, L., Gaussier, P.: Intuitive human robot interaction based on unintentional synchrony: A psycho-experimental study. In: Development and Learning and Epigenetic Robotics (ICDL), 2013 IEEE Third Joint International Conference on. pp. 1-7 (Aug 2013)

9. Hasnain, S.P., Mostafaoui, G., Gaussier, P.: A synchrony-based perspective for partner selection and attentional mechanism in human-robot interaction. Paladyn, Journal of Behavioral Robotics, Volume 3, Issue 3, Pages 156171 (2012)

10. Issartel, Marin, Cadopi: Unintended interpersonal co-ordination: "can we march to the beat of our own drum?". Neurosci Lett. 2007 Jan 16;411(3):174- (2007)

11. Jenkins, O.C., Mataric, M.J., Weber, S.: Primitive-based movement classification for humanoid imitation. In: Proceedings, First IEEE-RAS International Conference on Humanoid Robotics (Humanoids-2000). pp. 1-18 (2000)

12. Kay, Kelso, Saltzman: Space-time behaviour of single and bimanual rythmical movments. Journal of experimental Pysychology: Human perception and performance 13 (1987)

13. Kelso, J.A.S.: Haken-Kelso-Bunz model. Scholarpedia 3(10), 1612 (2008), revision 91336

14. Kilner, J., Paulignan, Y., Blakemore, S.: An interference effect of observed biological movement on action. Current Biology 13(6), 522 - 525 (2003)

15. Lagarde, J., Kelso, J.: Binding of movement, sound and touch: multimodal coordination dynamics. Experimental Brain Research 173(4), 673-688 (2006)

16. Mannella, F., Baldassarre, G.: Selection of cortical dynamics for motor behaviour by the basal ganglia. Biological Cybernetics 109(6), 575-595 (2015)

17. Pataleone, J.: Synchronization of metronomes. American Journal of Physics (2002)

18. Phillips-Silver, J., Aktipiz, A., Bryant., G.A.: The ecology of entrainment: Foundations of coordinated rhythmic movement. Music perception 28.1 (2010): 314. PMC. Web. 9 Sept. 2015. (2010)

19. Revel, A., Andry, P.: Emergence of sturctured interactions: From theoretical model to pragmatic robotics. Neural Networks vol. 22, (2009)

20. Terman, D., Wang, D.: Global competition and local cooperation in a network of neural oscillators. Physica D: Nonlinear Phenomena 81(1-2), 148 - 176 (1995)

21. Varlet, M., Marin, L., Issartel, J., Schmidt, R., Bardy, B.G.: Continuity of visual and auditory rhythms influences sensorimotor coordination. PloS one 7(9), DOI-10.1371/journal.pone, e44082 (2012)

22. Varlet, M., Marin, L., Raffard, S., Schmidt, R.C., Capdevielle, D., Boulenger, J.P., DelMonte, J., Bardy, B.G.: Impairments of social motor coordination in schizophrenia. PloS one 7(1), DOI-10.1371/journal.pone, e29772 (2012)

23. Wilson, H., J, J.C.: Excitatory and inhibitory interactions in localized populations of model neurons. Biophys. J., Vol. 12 (1972) 